\title{
LA «DESCONEXIÓN DIGITAL" A LOMOS DE LA SEGURIDAD Y SALUD EN EL TRABAJO
}

\author{
The «digital disconnect» on the back of occupational health and safety
}

Francisco Trujillo Pons*

Universidad de Valencia, España

\section{RESUMEN}

En la actuación preponderancia otorgada al teletrabajo o trabajo a distancia debido a la crisis sanitaria está empujando a difuminar la línea que separa el tiempo de trabajo efectivo del tiempo de ocio y descanso. A la postre, provoca prácticamente que el empleado nunca desconecte del trabajo. Y ello es así porque las Tecnologías de la Información y de la Comunicación (TIC) empujan a una disponibilidad horaria y locativa constante de los trabajadores, incluso, fuera de su horario laboral. No son de extrañar en este sentido, comunicaciones de jefes, compańeros de trabajo o, incluso, clientes a través de distintos medios como ahora: correos electrónicos que necesitan contestación urgente, llamadas telefónicas a horas intempestivas o, sobre todo en el sentido de este artículo, mediante el smartphone, bien por mensajes SMS — aunque es cierto que, cada vez, en más desuso — bien por mensajes a través de múltiples programas de mensajería instantánea (aplicaciones tipo WhatsApp, iMessage o Telegram). Sin olvidar tampoco que el smartphone cuenta con la posibilidad de incorporar cuentas de correo corporativos, lo que ańade un plus más a esta «hiperconectividad" y esa sensación urgente y fatigosa que provoca en el trabajador la contestación inmediata y sin esperas de toda comunicación que recibe. Este contexto, exige un estudio sobre las consecuencias negativas que pueden sufrir los trabajadores desde su seguridad y salud. Los riesgos psicosociales en este sentido han de ser valorados por las empresas que, por su parte, han de dar cumplimiento a su acción preventiva consagrada en la Ley 31/1995, de 8 de noviembre, de prevención de riesgos laborales.

Palabras clave: normativa, prevención de riesgos, teletrabajo, medidas, riesgos laborales, desconexión digital, hiperconectividad.

\section{ABSTRACT}

The preponderance given to teleworking or remote work due to the health crisis is pushing to blur the line that separates effective work time from leisure and rest time. In the end, it practically causes the employee to never disconnect from work. And this is so because Information and Communication Technologies (ICT) push workers to have a constant time and location availability, even outside their working hours. In this sense, communications from bosses, co-workers or even clients through different means like now are not surprising: emails that need an urgent answer, phone calls at untimely hours or, especially in the sense of this article, through the smartphone, either by SMS messages - although it is true that, every time, in more disuse - or by messages through multiple instant messaging programs (applications such as WhatsApp, iMessage or Telegram). Not forgetting either that the smartphone has the possibility of incorporating corporate email accounts, which adds one more plus to this "hyperconnectivity" and that urgent and tiring feeling that causes the worker to respond immediately and without waiting for any communication he receives. This context requires a study on the negative consequences that workers may suffer from their safety and health. Psychosocial risks in this sense must be assessed by companies which, for their part, must comply with their preventive action enshrined in Law 31/1995, of November 8 , on the prevention of occupational hazards.

Keywords: regulations, risk prevention, teleworking, measures, occupational risks, digital disconnection, hyperconnectivity

* Correspondencia a: Francisco Trujillo Pons. Avenida de los Naranjos, s/n, 46022, (Valencia), España. — francisco.trujillo@ uv.es - https: //orcid.org/0000-0002-1742-2037

Cómo citar: Trujillo Pons, Francisco. (2021). «La "desconexión digital” a lomos de la seguridad y salud en el trabajo»; Lan Harremanak, 45, 257-275. (https: //doi.org/10.1387/lan-harremanak.21955).

Recibido: 14 agosto, 2020; aceptado: 30 octubre, 2020.

ISSN 1575-7048 - elSSN 2444-5819 / (C) 2020 UPV/EHU

(c) (i) Esta obra está bajo una licencia

Creative Commons Atribución 4.0 Internacional 


\section{La «hiperconectividad» laboral potenciada por el COVID-19}

El total asentamiento de las conocidas "Tecnologías de la Información y la Comunicación» (en lo sucesivo, TIC $^{1}$ ) en la sociedad y, por extensión lógica en el ámbito laboral son beneficiosas en muchos sentidos. No en vano, según diversas teorías, las TIC se crearon para «liberar a las personas del trabajo tedioso, degradante y alienante y les permitirán satisfacer su curiosidad, aprender, disfrutar de la vida y dedicar la mayor parte de su tiempo al ocio y al juego" (Dosi y Virgillito, 2019: 8). No obstante, lo anterior, en otras ocasiones, las TIC pueden difuminar determinados derechos laborales consagrados en la normativa nacional, los cuales, han de ser garantizados siempre en su integridad y sin excepción.

La digitalización instaurada en el actual marco de las relaciones laborales provoca un uso masivo de las herramientas tecnológicas de uso profesional estricto y una "hiperconectividad» o conectividad constante del trabajador que pone en duda un derecho de vital importancia en el ámbito laboral: el derecho a la seguridad y salud. Máxime tras la actual preponderancia hacia el teletrabajo, provocado por la presencia del COVID-19. La pandemia mundial, con el régimen de confinamiento y las distintas fases vividas hasta la «nueva normalidad" ha obligado a muchos trabajadores a prestar los servicios «en remoto». En los momentos que vive el mercado laboral por la crisis sanitaria, el estrés que puede surgir en el uso de las TICs han de ser la excepción y no la regla común. Esta conectividad constante aboca al trabajador a no desconectar nunca de su trabajo, con las consecuencias perniciosas que ello puede provocar a su salud: las empresas han de evaluar, como obligadas merced a la Ley 31/1995, de 8 de noviembre, de prevención de Riesgos Laborales (BOE 10 nov. 1995, en adelante LPRL), los riesgos psicosociales del uso de las TICs en el ámbito laboral.

En efecto, estamos ante una nueva era, la era de la «interconectividad digital» por la que un grandísimo volumen de datos e información se encuentran en la conocida como «nube» que permiten modelos de trabajo interconectados en los que el jefe puede utilizar medios TICs para contactar digitalmente o a distancia con el empleado. Estos modelos de trabajo que, en la actualidad, por el régimen de confinamiento y preferencia dada al trabajo a distancia por la presencia del COVID-19, alcanzan su máxima expresión con el «teletrabajo» (o trabajo a distancia) según el cual, la prestación del trabajo se realiza precisamente con el uso de las TICs con la particularidad de que la ejecución del mismo se lleva a cabo, de modo regular, fuera de los locales de la empresa. Esta última característica lleva a diferenciar el «teletrabajo» del trabajo a distancia regulado en el artículo 13 del ET y, más recientemente, conforme al Real Decreto-Ley 28/2020, de 22 de septiembre, de trabajo a distancia

\footnotetext{
1 Estas son, todas aquellas herramientas relacionadas con la transmisión, procesamiento y almacenamiento digitalizado de la información.
} 
(en lo sucesivo, RDLTD BOE, 23 sept. 2020) que reincide en la ley orgánica de protección de datos regulando el derecho a la desconexión digital (ex. art. 18): en efecto, la ejecución del trabajo, que podría realizarse igualmente en los locales del empleador, se efectúa fuera de éstos de manera regular mientras que, en el trabajo a domicilio, la prestación de trabajo se ejecuta en el domicilio del trabajador o en el lugar libremente elegido por éste y sin vigilancia del empresario. En consecuencia, en el "teletrabajo», la empresa dentro de sus poderes de dirección ${ }^{2}$, puede controlar y vigilar al trabajador (por ejemplo, a través de programas informáticos que registran los movimientos del trabajador en el ordenador ${ }^{3}$.

\section{La «desconexión digital» en el ámbito laboral}

El exceso de jornada laboral que están viviendo muchos trabajadores en España está protegido por el actual derecho a la «desconexión digital» en el ámbito laboral tratado en abundancia por doctrina iuslaboralista (entre otras muchas obras: Altés, y Yagüe, 2019; Vidal, 2018 y; Biurrun, 2018); derecho que ha de ser tratado de forma conjunta por su relación directa con la seguridad y salud en el trabajo. Derecho de índole digital que entró en vigor en España el 7 de diciembre de 2018, tomando forma en la Ley Orgánica 3/2018, de 5 de diciembre, de Protección de Datos Personales y Garantía de los Derechos Digitales (BOE 6 dic., en adelante LOPDPGDD), por la que se modifica, entre otras disposiciones legales, el Texto Refundido de la Ley del Estatuto de los Trabajadores (ET), mediante la inclusión de un nuevo artículo, el 20 bis. Se reconoce así en España, desde hace apenas dos años, el derecho a la "desconexión digital» en el trabajo, en el marco del derecho a la intimidad en el uso de dispositivos digitales en el ámbito laboral. En este sentido, de acuerdo al artículo 88 de la citada Ley Orgánica, la empresa tiene la obligación de establecer una política interna sobre las modalidades del ejercicio del derecho a la "desconexión digital», previa audiencia de los representantes de las personas trabajadoras. Conforme al mismo, el trabajador fuera del tiempo de trabajo legal o convencionalmente establecido no tiene la obligación de responder a llamadas, correos electrónicos corporativos, ni mensajes de texto. De ponerse en la práctica de la empresa (presencial o a distancia) el ejercicio de este derecho, la carga mental y la fatiga informática del trabajador no tendrían los niveles necesarios para repercutir en la salud de los trabajadores.

2 Consultar: Pérez de los Cobos y Orihuel, Francisco (2019): «Poderes del empresario y derechos digitales del trabajador». Trabajo y derecho: nueva revista de actualidad y relaciones laborales, ISSN 2386-8090, N. ${ }^{\circ} .59$.

3 Consultar: López Lourdes (2018): «Vigilar ocultamente a los trabajadores en la empresa y la reciente doctrina del Tribunal Europeo de Derechos Humanos». Disponible en: https: //www.ga-p. $\mathrm{com} / \mathrm{wp}$-content/uploads/2018/07/vigilar-ocultamente-a-los-trabajadores-en-la-empresa-y-la-reciente-doctrina-del-tribunal-europeo-de-derechos-humanos.pdf (Accedido: 3-06-2020). 
No obstante lo anterior, en la práctica, muchas empresas incumplen de manera sistemática el derecho, luego, urge un desarrollo reglamentario ${ }^{4}$ de la actual normativa que lo regula, esta es: la LOPDPGDD de cuyo contenido, destaca en este sentido su artículo 88, dedicado en sus tres apartados al derecho a la «desconexión digital» en el trabajo así como, igualmente, el señalado artículo 18 del reciente RDLTD. Partiendo de esta premisa, es indudable la necesidad de una regulación más amplia del derecho a la "desconexión digital» en el trabajo; es un reto que marca la sociedad y que el legislador ha de tomarlo como tal; un derecho incipiente (apenas no llega a dos años de su existencia en el ordenamiento jurídico español) que está llamado, en un futuro cercano, a ocupar una posición nuclear en las relaciones laborales.

\section{La seguridad y salud en el trabajo en el marco del trabajo a distancia}

Merece especial atención por su trascendencia para la salud del trabajador que tiene imposibilitado desconectar, hacer hincapié en la prevención de riesgos laborales, vista desde la óptica de la prestación a servicios a distancia o «en remoto".

La globalización del mercado laboral y el aumento de la competitividad ha ido provocando, en los últimos ańos, una mayor flexibilidad en la organización del tiempo y una nueva forma de entender la conectividad. En este sentido, las formas de organización tradicionales han pasado a ser más tecnológicas. Como recoge la Nota Técnica Preventiva 1122 (NTP 1122) del Instituto Nacional de Seguridad y Salud en el Trabajo (INSST), todo ello viene provocado debido a la "cotidianidad en el uso de las TIC en el ámbito laboral» (Santamaría, 2018: 2). Estas formas de trabajo más flexibles y tecnológicas son una tendencia al alza en los próximos años y es necesario una ley garantista que proteja a los trabajadores sobre el uso excesivo de los medios tecnológicos laborales, como ahora, el ordenador, tablets o el teléfono móvil inteligente (smartphone), que se engloban en las TICs.

Según señala acertadamente la doctrina iuslaboralista ${ }^{5}$, todo ello adquiere una dimensión mucho más grande cuando las clásicas unidades físicas de refe-

\footnotetext{
4 Consultar: Zamora, Santiago (2019): «Desconexión digital ¿novedad o anécdota?», Diario La Ley, ISSN 1989-6913, N. 9363.

5 Entre otros, ampliamente: Altés, Juan Antonio y Yagüe, Sergio (2020): «A vueltas con la desconexión digital: eficacia y garantías de lege lata». Labos, Vol. 1, No. 2, pp. 61-87 / doi: https: //doi. org/10.20318/labos.2020.5539 EISSN 2660-7360 - http: //www.uc3m.es/labos; Molina, Cristóbal (2017): "Jornada Laboral y Tecnologías de la Infocomunicación: «desconexión digital», garantía al descanso». Temas laborales, núm. 138/2017; Todolí, Adrián (2017): «El derecho a la desconexión digital ya existe. Nos venden humo si no se establecen sanciones claras para quien lo vulnere». Disponible en: https: //adriantodoli.com/2017/03/29/el-derecho-a-la-desconexion-digital-ya-existe-nos-
} 
rencia (centros de trabajo «analógicos»), tienden a devenir en virtuales. En España no es de extrañar que existan evidencias de que se trabajen horas fuera de la jornada laboral desde los dispositivos electrónicos, especialmente en las empresas del sector tecnológico. Más si se trata de empresas del sector tecnológico. En un estudio queda constatado que en las empresas de este sector en donde el acceso a los dispositivos tecnológicos es sencillo, la mayoría de sus empleados reconocen usarlos para trabajar fuera de su jornada laboral ${ }^{6}$.

Este sector ya de por sí funciona de forma algo diferente ya que los horarios son más flexibles pero las jornadas de trabajo raramente bajan de 10 horas diarias. Puede pensarse en empresas «tipo Google», horarios flexibles, ambiente agradable de trabajo, todas las comodidades imaginables en la empresa y, sin embargo, pocos de sus empleados pueden conciliar su vida laboral con la familiar, de hecho, el perfil del empleado de este tipo de empresas es una persona joven con pocas o ninguna carga familiar que dedica todo su tiempo al trabajo.

La aparición del citado RDLTD ha de servir para alcanzar un ejercicio del «teletrabajo» más equilibrado. Si se acude al RDLTD, en su artículo 16 (dedicado a la evaluación de riesgos y planificación de la actividad preventiva) se regula la obligatoriedad empresarial de evaluar los riesgos y planificar la actividad preventiva del trabajo a distancia y tener en cuenta, sobremanera, los riesgos característicos de esta modalidad de trabajo, poniendo especial atención en los factores psicosociales, ergonómicos y organizativos. En particular, deberá tenerse en cuenta la distribución de la jornada, los tiempos de disponibilidad y la garantía de los descansos y desconexiones durante la jornada. Igualmente, continúa señalando el precepto:

La evaluación de riesgos únicamente debe alcanzar a la zona habilitada para la prestación de servicios, no extendiéndose al resto de zonas de la vivienda o del lugar elegido para el desarrollo del trabajo a distancia.

En este mismo sentido, continúa señalando que:

La empresa deberá obtener toda la información acerca de los riesgos a los que está expuesta la persona que trabaja a distancia mediante una metodología que ofrezca confianza respecto de sus resultados, y prever las medidas de protección que resulten más adecuadas en cada caso.

venden-humo-si-no-se-establecen-sanciones-claras-para-quien-lo-incumpla/ (Accedido: 2-6-2020) y; Trujillo Pons, Francisco (2020): «La «desconexión digital» en el ámbito laboral». Colección Laboral núm. 264, Tirant lo Blanch, Valencia. 166 páginas. 25 septiembre 2020. ISBN: 978-84-1355912-4.

6 Consultar: Rodríguez Fernández, María Luz y Pérez del Prado, Daniel (2017). «Economía digital: Su impacto sobre las condiciones de trabajo y empleo. Estudio de caso sobre dos empresas de base tecnológica». Disponible en: http: //fdialogosocial.org/public/upload/2/23_FdS_Economia-digital-impacto-condiciones-trabajo-y-empleo_2017_final.pdf (Accedido: 2-6-2020). 
Más, la LPRL se aplica a todos los trabajadores por cuenta ajena (art. 3) y sea cual sea el lugar en el que se preste servicios; desde el hogar también existen riesgos laborales que hay que considerar debidamente. Riesgos tales como como la luminosidad, los dolores musculoesqueléticos o la fatiga mental y física han de ser evaluados por los empleadores conforme a su obligación de proteger la seguridad y salud de sus trabajadores. En este sentido pues, son de significar las disciplinas de la prevención de riesgos laborales que estudian la psicosociología y la ergonomía del trabajo.

Con la «hiperconectividad» constante del trabajador, se incrementan los riesgos relativos a la fatiga informática que puede tener consecuencias para el trabajador tanto del plano físico como del plano mental ${ }^{7}$. Surge desde este prisma el "tecnoestrés»" ${ }^{8}$ el cual, se materializa cuando la gestión de la empresa no considera a las herramientas tecnológicas como potenciales estresores para sus trabajadores; como consecuencia del mismo son comunes episodios de fatiga (física y mental), dolores de cabeza, ansiedad e incluso trastornos musculoesqueléticos. Irremediablemente, la «hiperconectividad» digital y la salud laboral son dos cuestiones que van unidas'; son necesarias mejoras sustanciales en la previsión de la salud del trabajador, en el señalado sentido de la susceptibilidad por exceso ante un abrumador horario de trabajo, como adicionalmente, la carencia de apoyo social que, en suma, puede desembocar en episodios de estrés y otros trastornos. Para evitar la materialización de estos estresores, la empresa ha de adoptar medidas preventivas, por ejemplo, por medio de evaluaciones de riesgos psicosociales (pueden servir, como recoge el señalado RDLTD, cuestionarios ${ }^{10}$ o check-lists) o

7 Sobre este tema, CCOO Endesa a fecha de 18 de mayo de 2020, con motivo de la situación de la pandemia mundial redactó una carta de intenciones indicando de la necesidad de "informar y formar a las personas trabajadoras sobre higiene, escritorio, el uso correcto de las pantallas de visualización, los riesgos higiénicos (iluminación, condiciones de confort térmico, ruido, etc.), los riesgos ergonómicos (trastornos musculo esqueléticos), otros riesgos como los electromagnéticos, la fatiga visual, la fatiga física, la fatiga informática y la fatiga mental, así como el estrés tecnológico y la conexión digital continuada». CCOO Endesa (2020).

${ }^{8}$ El concepto de tecnoestrés está directamente relacionado con los efectos psicosociales negativos del uso de las Tecnologías de la información y la comunicación.

9 Consultar: Domingo, José y Salvador, Neus (2020): «Hiperconectividad digital y salud laboral». Diario La Ley, ISSN 1989-6913, N.o 9638.

${ }_{10}$ Pueden servir a modo práctico tanto el introducido a nivel estatal por el Gobierno a fecha de 17 de marzo dirigido a controlar a aquellos trabajadores que, con motivo del COVID-19 se vieron obligados a teletrabajar. El mismo recaba información de las características específicas del lugar del domicilio en el que la persona desempeńa su labor con la ayuda del ordenador y elementos auxiliares (teléfono, documentos, etc.). A todas luces resulta insuficiente por no incorporar medidas relativas a la "desconexión digital". Ahora bien, ha de verse como un listado no exhaustivo y por tanto, como una identificación de las condiciones que, como mínimo, debería tener el puesto de "teletrabajo». Disponible en: http: //pruebas.croem.es/Web20/CROEMPrevencionRiesgos.nsf/67179F5C2C9B5 6DEC1258536002ECEEB/\$FILE/cuestionario\%20autoevaluaci\%C2\%B4n\%20teletrabajo.pdf (Accedido a 26-6-2020]. Como también, a nivel de Comunidad Valenciana, establecido por medio del DECRETO 82/2016, de 8 de julio, del Consell, por el que se regula la prestación de servicios en ré- 
bien, dando participación a los trabajadores para que sean ellos los que participen en la introducción de las nuevas tecnologías para que la adaptación sea así y no al revés (que los trabajadores se amolden a las TICs).

Más en este sentido, se han de adoptar una serie de medidas y prácticas a través de sus responsables en prevención de riesgos laborales, por ejemplo, que los trabajadores, estén donde estén realicen pausas desde sus dispositivos digitales en aras a conseguir un descanso de pantalla y, de este modo, minimizar el riesgo de fatiga visual. Del mismo modo, dar autonomía al trabajador para que pueda ponerse un horario determinado que le permita gestionar las pausas, así como las horas de comida. Con este horario determinado, el trabajador será capaz de apagar los dispositivos digitales (teléfono móvil u ordenador) y así ejercitar con plenas garantías su derecho a la "desconexión digital» y, obviamente no sufrir el estrés y fatiga de tener que contestar llamadas y mensajes fuera del horario laboral.

Desde el plano de la ergonomía laboral, existe una norma de desarrollo reglamento de la LPRL a considerar: el Real Decreto 488/1997, de 14 de abril, sobre disposiciones mínimas de seguridad y salud relativas al trabajo con equipos que incluyen pantallas de visualización (BOE 23 abr. 1997) por medio del cual, se fijan las medidas mínimas que deben adoptarse para la adecuada protección de los trabajadores, entre las que destacan las destinadas a garantizar que de la utilización de los equipos que incluyen pantallas de visualización de datos (PVDs $\left.{ }^{11}\right)$ por los trabajadores no se deriven riesgos para la seguridad y salud de los mismos. Según el Real Decreto, están excluidos de su ámbito de aplicación «los sistemas llamados portátiles, siempre y cuando no se utilicen de modo continuado en un puesto de trabajo» (art. 1.3 apdo. d). No obstante, el trabajador que utiliza asiduamente un ordenador o un smartphone con fines estrictamente profesionales debería tener protección conforme a esta normativa. De modo que el empresario, como garante de la seguridad de sus trabajadores, ha de adoptar las medidas necesarias para que la utilización por los trabajadores de equipos con pantallas de visualización no suponga riesgos para su seguridad o salud o, si ello no fuera posible, para que tales riesgos se reduzcan al mínimo (art. 3). A estos efectos, debe evaluar los riesgos para

gimen de teletrabajo del personal empleado público de la Administración de la Generalitat. DOGV núm. 7828 de 14.07.2016. Recuperado de: https: //www.dogv.gva.es/es/eli/es-vc/d/2016/07/08/82/ dof/vci-spa/pdf (Accedido a 26-062020), el cual sí que determina una pregunta a contestar por el «teletrabajadonः: «¿Desconecta los aparatos eléctricos cuando no los está utilizando?».

${ }^{11}$ De acuerdo con su art. 2 se define como "pantalla alfanumérica o gráfica, independientemente del método de representación visual utilizado». Siendo el puesto de trabajo aplicable a esta normativa el constituido por un equipo con pantalla de visualización provisto, en su caso, de un teclado o dispositivo de adquisición de datos, de un programa para la interconexión persona/máquina, de accesorios ofimáticos y de un asiento y mesa o superficie de trabajo, así como el entorno laboral inmediato. Y, el trabajador, como aquel que habitualmente y durante una parte relevante de su trabajo normal utilice un equipo con pantalla de visualización. Como se observa, entronca con el derecho a la «desconexión digital» en el trabajo. 
la seguridad y salud de los trabajadores, teniendo en cuenta en particular los posibles riesgos para la vista y los problemas físicos y de carga mental, así como el posible efecto añadido o combinado de los mismos. Indudablemente los problemas físicos y mentales se pueden derivar tras una conectividad constante de los dispositivos digitales. La evaluación que ha de realizarse por el empresario, se llevará a cabo tomando en consideración las características propias del puesto de trabajo (atendiendo a la naturaleza de la relación laboral y también sin desdeñar el puesto de los altos directivos) y las exigencias de la tarea y entre éstas, especialmente, las siguientes: a) el tiempo promedio de utilización diaria del equipo; b) el tiempo máximo de atención continua a la pantalla requerido por la tarea habitual; c) el grado de atención que exija dicha tarea. En suma, con el cumplimiento de esta normativa, y en línea directa con la garantía del ejercicio saludable de desconectar de la tecnología laboral, los trabajadores contarán con una evaluación de los dispositivos digitales que habitualmente utilizan en el trabajo y no sufrirán desgastes en términos físicos o mentales por la sobrecarga de un uso excesivo.

Igualmente, en términos de prevención de riesgos laborales del trabajador en remoto, a nivel público de la Comunidad Valenciana, conviene reseñar el citado DECRETO 82/2016, de 8 de julio, del Consell, por el que se regula la prestación de servicios en régimen de "teletrabajo» del personal empleado público de la Administración de la Generalitat (DOGV núm. 7828 de 14.07.2016) pues, su artículo 9 ("Seguridad y salud en el trabajo») determina que se deberá verificar previo al inicio de la prestación que la realización del cuestionario no supone riesgo para la salud del «teletrabajadon. Ello no obsta, como sigue recogiendo, que el órgano competente en materia preventiva a nivel de la Comunidad Valenciana, realice una inspección domiciliaria a fin de comprobar las condiciones alegadas en el citado cuestionario. Las condiciones del puesto de trabajo también podrán ser objeto de revisión y análisis durante toda la vigencia del programa, previa comunicación a la persona interesada.

Todo ello, tiene respaldo normativo en el ordenamiento jurídico español en atención al artículo 4.2 del Real Decreto 39/1997, de 17 de enero, por el que se aprueba el Reglamento de los Servicios de Prevención (BOE 31/01/1997) ya prevé esta situación en el contenido general de evaluación. Así:

A partir de dicha evaluación inicial, deberán volver a evaluarse los puestos de trabajo que puedan verse afectados por: a) La elección de equipos de trabajo, sustancias o preparados químicos, la introducción de nuevas tecnologías o la modificación en el acondicionamiento de los lugares de trabajo.

La voluntad del propio trabajador respeto a la obligación de no conectarse fuera de su jornada laboral es esencial para que el derecho a su desconexión tecnológica del trabajo se ejecute. Ha de ser consciente de los riesgos laborales a los que se puede enfrentar por no descansar y permanecer conectado ininterrumpidamente. 
Daños consecuencia de esta falta de «desenganche» pueden ser, dolores de espalda y cuello, problemas de visión y audición, e incluso, alteraciones del sueño y cognitivas. A ello, se le pueden sumar conductas sedentarias y de aislamiento que, puede acabar generando dependencia, adicción y ansiedad: cada vez más personas padecen nomofobia ${ }^{12}$, un miedo irracional a no tener a mano su smartphone $e^{13}$.

En efecto, en períodos de descanso, como en vacaciones, antes de la inmersión de las TIC en la sociedad, los trabajadores no llevaban consigo sus teléfonos móviles o portátiles pero, al llevarlo siempre encima e incluso, al montar la propia oficina en casa en supuestos de modalidades de teletrabajo, resulta muy complicado desconectar digitalmente (pueden sentirse incentivos e incluso forzados a trabajar aun cuando no deberían). La desconexión es necesaria en términos de salud: mejoran los niveles de bienestar, felicidad y satisfacción personal, así como en los estados de depresión y ansiedad ${ }^{14}$. Algunos consejos para lograr el descanso absoluto fuera de la jornada laboral pueden ser: 1) dejar el equipo operativo, si está en la oficina, o compartir los documentos y archivos importantes en la nube o en carpetas compartidas a través de una conexión VPN;2) programar el correo electrónico con una respuesta automática informando de que no se está operativo en estos momentos e indicando que pueden contactar con otro compañero si lo necesitan y; 3) desactivar las notificaciones de las herramientas de trabajo. Consejos, en definitiva, que pueden ser utilizados no solo para los trabajadores sino también para superiores jerárquicos que como líderes de equipos cuentan con trabajadores a su cargo pueden ser los siguientes.

Inexcusablemente, la seguridad y salud en el teletrabajo tiene una relación directa con la aparición de riesgos psicosociales ${ }^{15}$. En esta línea, la citada NTP 1122

12 El término nomofobia ha sido un término definido como el mido al estar sin teléfono móvil. Proviene de «no-mobile-phone phobia» es decir: la dependencia en grado sumo al teléfono móvil.

13 Consultar: Sánchez Lacuesta, Javier (2020), «Desconexión digital». Disponible en: http: //www. biomecanicamente.org/news/ibv/item/1809-levante-articulo-abril2020.html (Accedido a 5-10-2020).

14 Estos beneficios han sido demostrados en múltiples estudios, por ejemplo, el llevado a cabo por las universidades de Stanford y Nueva York. En su estudio "The Welfare Effects of Social Media» llevado a cabo por los investigadores Hunt Allcott, Luca Braghieri, Sarah Eichmeyer, and Matthew Gentzkow (8 nov. 2019) se observó cómo miles de usuarios de la red social Facebook que desactivaron sus cuentas durante un mes, demostraron evidentes mejoras en su salud psicosocial. Recuperado de (inglés): http: //web.stanford.edu/ - gentzkow/research/facebook.pdf [Consulta a 5 oct. 2020].

15 Alegre, Manuel (2018): La prevención de los nuevos riesgos psicosociales y el derecho a la «desconexión digital». Gestión práctica de riesgos laborales: Integración y desarrollo de la gestión de la prevención, ISSN 1698-6881, N. ${ }^{\circ}$ 155; Mińarro Yanini, Margarita (2020). "El sistema de prevención de riesgos laborales como garantía de efectividad del derecho a la desconexión digital", en Rodríguez-Piñeiro Royo, M. y Todolí Signes, A. (eds.) Vigilancia y control en el Derecho del Trabajo Digital. Cizur Menor (Navarra): Aranzadi-Thomson Reuters, pp. 577-596; Arrieta, Francisco Javier (2019): La desconexión digital y el registro de la jornada diaria en Espańa como mecanismos para garantizar el descanso, la salud y el bienestar de los trabajadores digitales a distancia. Lan harremanak: Revista de relaciones laborales, ISSN 1575-7048, N.o 42, 2019 (Ejemplar dedicado a: Digitalización, conciliación y trabajo como mercancía/ Digitalizazioa, uztartzea eta lana merkatalgai gisa). 
incorpora un listado no exhaustivo de factores de riesgo psicosocial por el uso inadecuado y no planificado de las TIC's relacionados con el tiempo de trabajo:

- Prolongación de la jornada laboral (antes de su comienzo o al finalizar la misma) y realización del trabajo en horarios inusuales (noche, fin de semana etc.) debido a la conectividad permanente y al uso inadecuado de las TICs.

— Dificultad de conciliar la vida familiar y laboral.

- Aumento de las interrupciones en el desarrollo del trabajo, en especial en el uso del correo electrónico.

- Tareas no previstas y multitarea que pueden prolongar la jornada laboral.

— Realización de tareas en "periodos de espera» o "tiempos muertos» (entre desplazamientos y/o viajes) que antes eran «inactivos» (ya que no se podía realizar el trabajo sin estar presente en la oficina) y ahora son trabajados y pueden suponer un añadido al tiempo de trabajo de la jornada laboral.

- Asincronismo temporal para interactuar con otros trabajadores ubicados en países con diferentes franjas horarias, lo que incide en la jornada laboral, prolongando o adelantando el comienzo de la misma.

- Cultura de empresa sin una política clara sobre promoción de trabajadores remotos, lo que hace aumentar la competitividad, pudiendo ocasionar un aumento de las horas de trabajo usando las TICs fuera del horario laboral y del centro de trabajo, ocasionando a su vez prácticas laborales inadecuadas como el leaveism (entendido en su acepción de realización del trabajo en el hogar, fuera de la jornada laboral, para adelantar trabajo sin dar parte de esta práctica ni a compańeros ni a superiores jerárquicos).

- Inestabilidad laboral y ausencia de cultura empresarial clara entorno al uso inadecuado de las TICs fuera de la jornada laboral como el leaveism (entendido en su acepción de llevar el trabajo "al día» y realizarlo fuera de la jornada laboral para lograr consolidar el puesto de trabajo ante la amenaza de una no renovación o despido).

- Cultura empresarial que no tenga en cuenta la movilidad física constante para la realización del trabajo (a nivel local, autonómico, nacional o internacional en países con o sin diferentes franjas horarias) como tiempo de trabajo y/o la prolongación de la jornada laboral que ello pueda suponer.

La salud, según la Organización Mundial de la Salud (OMS), está definida como «un estado de completo bienestar físico, mental y social, y no solamente la ausencia de afecciones o enfermedades ${ }^{16}$ ", por consiguiente, no es sólo física sino

16 La cita procede del Preámbulo de la Constitución de la OMS, que fue adoptada por la Conferencia Sanitaria Internacional, celebrada en Nueva York del 19 de junio al 22 de julio de 1946, firmada el 22 de julio de 1946 por los representantes de 61 Estados (Official Records of the World Health Organization, N. ${ }^{\circ}$ 2, p. 100), y entró en vigor el 7 de abril de 1948. La definición no ha sido modificada desde 1948. 
también mental, se abre un debate acerca de si que la empresa mantenga el contacto digitalmente con el trabajador y que éste tenga que realizar trabajo fuera de su jornada laboral afecta negativamente a su salud psicosocial. Ya no sólo que tenga que realizar trabajo a deshoras, también el hecho de que tenga que estar disponible, dedicando parte de su atención, aunque no tenga que trabajar puede contribuir a generarle estrés y empeorar su salud general.

No ha de olvidarse que merced a la doctrina constitucional, la protección eficaz de la salud de los trabajadores como eje central de la LPRL, incluye la protección y tutela frente a los riesgos psicosociales (STCO 62/2007, de 27 de marzo). No solo el Tribunal Constitucional también destaca jurisprudencia del TJCE (15 de noviembre de 2001, C-49/00), al igual que el Tribunal Supremo (STS 101/2016, 16 de febrero). En consecuencia, la evaluación de riesgos laborales ha de incluir posibles factores estresantes que, a nuestro interés, son obvios desde el punto de vista de la sobrecarga digital. No obstante, la determinación de enfermedad profesional no es así, pues según Real Decreto 1299/2006, de 10 de noviembre, por el que se aprueba el cuadro de enfermedades profesionales en el sistema de la Seguridad Social y se establecen criterios para su notificación y registro (BOE 19 diciembre) hoy en día, no da cabida a los riesgos psicosociales. Según su texto, solamente tienen consideración como enfermedad profesional aquellas contraídas como consecuencia del trabajo ejecutado por cuenta ajena en las actividades que se especifiquen en el citado cuadro y que estén provocadas por elementos o sustancias que en el mismo se indiquen para cada enfermedad profesional. Lo cierto es que este cuadro legal ha de modificarse y ajustarse a esta nueva era digital; el uso normalizado de las TICs a través del cual resulta muy difícil separar la línea de la relación laboral de la extralaboral (familiares, amistades, ocio, etc.) ha de ser considerado en un futuro como causa de enfermedad profesional.

Por tanto, un trabajador que lleva mucho tiempo sin desconectar digitalmente, puede sufrir una excesiva carga mental que provoca insomnio, irritabilidad, mal humor, desmotivación, agotamiento mental, falta de energía y menor rendimiento. En efecto, según el INSST, cuando el trabajo exige una concentración, un esfuerzo de atención prolongado, etc., a los que el trabajador no puede adaptarse, y de los cuales no se puede recuperar, se habla ya de un estado de fatiga prolongada o fatiga crónica. Según sigue detallando el informe ${ }^{17}$ este tipo de fatiga, que ya no se recupera por el simple descanso, tiene consecuencias psicosociales para el trabajador mucho más serias que la fatiga normal, consecuencias orgánicas, físicas y psicosomáticas, tales como irritabilidad, depresión, falta

17 Consultar: Sebastián Olga y Del Hoyo, María Ángeles (2016): «La carga mental del trabajo». Instituto Nacional de Seguridad e Higiene en el Trabajo. Composición e impresión: Servicio de Ediciones y Publicaciones. INSHT Madrid ISBN: 84-7425-605-4, pág. 16. Disponible en: https: //www.insst.es/documents/94886/96076/carga+mental+de+trabajo/2fd91b55-f191-4779-be4f-2c893c2ffe37 (Accedido a 2-6--2020]. 
de energía y de voluntad para trabajar, salud más frágil, dolores de cabeza, mareos, insomnio, pérdida de apetito, etc., que probablemente no se sentirán sólo durante el trabajo o al finalizarlo, sino que a veces perduran y se notan incluso al levantarse de la cama, antes de ir a trabajar.

Urge a efectos de erradicar dicha carga mental, diseñar correctamente el puesto de trabajo. Así, merced a la obligación preventiva de las empresas (ex. art. 15 LPRL y ex. art. 16 RDLTD), la acción ha de ir encaminada a diseñar puestos de trabajo alejados de situaciones estresantes, organizando los tiempos de trabajo, dando descansos, pausas, flexibilizando, en definitiva, la jornada laboral, se habrá dado un paso importante para conseguir el menor atisbo de carga mental a los trabajadores. En estos casos, esta sobrecarga excesiva en la conectividad digital que padece el trabajador, puede tener tutela en forma de prestación laboral de la Seguridad Social, pero en forma de accidente de trabajo. De tal forma que, yendo al Real Decreto Legislativo 8/2015, de 30 de octubre, por el que se aprueba el texto refundido de la Ley General de la Seguridad Social (en adelante, LGSS. BOE 31 oct.) en su artículo 156 se entiende por accidente de trabajo: «toda lesión corporal que el trabajador sufra con ocasión o por consecuencia del trabajo que ejecute por cuenta ajena». Por consiguiente, al referirse a "toda lesión" se entiende que pueden ser lesiones tanto físicas como psíquicas, de ahí que las patologías que sufra el trabajador debido a esta situación puedan llegar a ser considerada como accidente de trabajo. En el artículo 156.3 la LGSS presume que dicha dolencia es accidente de trabajo, siempre y cuando se produzca durante el tiempo y en el lugar de trabajo (el «teletrabajo», aunque sea fuera de las instalaciones son a plenos efectos "el lugar de trabajo». Por otro lado, también cabe la posibilidad de que sean consideradas accidente de trabajo ciertas enfermedades que contraiga el trabajador con motivo de la realización de su trabajo, siempre que se pruebe que la enfermedad tuvo por causa exclusiva la ejecución del mismo [art. 156.2 e), LGSS]. En caso de ser considerada está situación como accidente de trabajo, se derivan consecuencias para la víctima o sus causahabientes, pues aparte de las prestaciones que se determinan legal o reglamentariamente, aparece la responsabilidad del pago del recargo de prestaciones económicas por accidentes laborales; pago que recaerá directamente sobre el empresario. Esta responsabilidad empresarial que entra en juego cuando fracasa la actividad preventiva, se encuentra regulada conforme al artículo 164.1 de la LGSS, según el cual: todas las prestaciones económicas que tengan su causa en accidente de trabajo, se aumentarán, en función de la gravedad de la falta, de un 30 a un $50 \%$, cuando la lesión se produzca, entre otros motivos, por inobservancia de las medidas generales o particulares de seguridad e higiene en el trabajo, o las elementales de salubridad. Para ello se exige la existencia de un comportamiento, doloso o culposo, del empresario.

Por lo que concierne a la conectividad constante del trabajador, en estos casos, la empresa podría ser considerada responsable de la enfermedad del trabaja- 
dor si, por ejemplo, este demostrara que fuera de su jornada recibe de forma indiscriminada llamadas telefónicas o correos electrónicos y, además, se le urge a responderlos.

Jurisprudencialmente, puede servir de ejemplo para estos casos la STSJ Cataluña [(Sala de lo Social, Sección 1.a), núm. 6827/2013 de 22 octubre (rec. núm. 7237/2012)] que declaró la procedencia para el recargo de prestaciones por accidente de trabajo, debido a una situación de acoso moral en el que existió omisión del empresario en su obligación de velar por la salud de la trabajadora, al tolerar situaciones organizativas, funcionales, directivas y ambientales, diferenciadas y específicas, que le afectaron, dando lugar a una grave patología psiquiátrica en la operaria. Es un ejemplo muy significativo porque, digitalmente en tiempos de COVID-19, también puede darse el acoso laboral. No en vano, existe un término que define el mismo como "ciberacoso en el trabajo» (network mobbing) y que ha sido tratado por la doctrina iuslaboralista ${ }^{18}$. A través del cual, el trabajador puede sufrirlo, por ejemplo, mediante el teléfono móvil a través de programas de mensajería. En estos casos se hace más que necesaria la desconexión fuera del horario del trabajo por lo que son totalmente inaceptables -salvo casos de fuerza mayor- no solo mensajes con contenido laboral sino, obviamente, también con contenido vejatorio u hostigador. En caso de demostrarse este hostigamiento digital, el caso de que el accidente de trabajo implique la baja médica, el trabajador tiene derecho a la prestación por incapacidad temporal. En cuanto a la indemnización por daños y perjuicios, si el accidente se produjo en su totalidad o en parte por omisión de medidas de seguridad, el trabajador podrá reclamar una indemnización por accidente laboral. Si la culpa del accidente de trabajo no pesa sobre el trabajador, sino que se puede demostrar que el empresario no puso todas las medidas de seguridad exigidas en la LPRL, el trabajador podrá reclamar a la empresa la indemnización que, por derecho, le corresponde. En suma, el acoso laboral, cuando genera una patología asociada a las prestaciones de Seguridad Social, será considerada como contingencia profesional en tanto que la causa principal ha sido derivada de la realización del trabajo.

Más, una de las consecuencias psicosociales de las más preocupantes si no la que más preocupa, es el síndrome conocido como síndrome del trabajador quemado (también conocido como «desgaste emocional» o, más comúnmente bajo el anglicismo burnout. Pese a que el estrés y el desgaste emocional son patologías laborales muy presentes en la actualidad, los tribunales españoles muestran un celo evidente en sus pronunciamientos. Es una circunstancia con una connota-

18 Ampliamente: Molina, Cristóbal (2019): «Del acoso moral («mobbing») al ciberacoso en el trabajo («network mobbing») viejas y nuevas formas de violencia laboral como riesgo psicosocial en la reciente doctrina judicial». Estudios financieros. Revista de trabajo y seguridad social: Comentarios, casos prácticos : recursos humanos, ISSN 1138-9532, N. ${ }^{\circ}$. 437-438, 2019, págs. 143-165. 
ción importante pues, en términos de eventuales incumplimientos del empresario como obligado conforme a la LPRL, se podría incrementar la sanción para el incumplidor al inmiscuirse el riesgo psicosocial del trabajador. Ello es así, debido al ya analizado recargo de prestaciones a la Seguridad Social que supone conforme al artículo $164^{19}$ de la LGSS, un aumento de la cuantía de todas las prestaciones económica que derivan de un accidente o enfermedad profesional del que debe hacerse cargo el empresario.

Asentado lo anterior, el burnout surgió en Estados Unidos a mediados de los años 70 como explicación a una respuesta de las personas al estrés laboral crónico. En sus inicios se denominaba de esta forma sólo a las consecuencias del estrés laboral de las personas que trabajaban dando servicio a personas, como por ejemplo enfermeras, médicos o profesionales de la enseñanza; pero con el tiempo se vio que los síntomas eran muy similares en personas con otro tipo de empleos por lo que se consideró una respuesta general al estrés laboral. Actualmente se define como la respuesta de las personas al estrés laboral crónico, cuando las estrategias de afrontamiento que se emplean para el estrés fallan o ya no resultan suficientes para hacer frente a la situación por la que pasa en empleado.

Los síntomas del síndrome son muy variados, uno de los más significativos es agotamiento emocional. Los trabajadores sienten que ya no pueden dar más de sí mismos, sienten que se han agotado su energía y recursos emocionales. Otro síntoma que aparece en las personas que padecen síndrome de burnout es bajos niveles de realización personal en el trabajo. Los empleados se sienten descontentos con su labor, con sus resultados; tienden a evaluarse negativamente y esa misma autoevaluación negativa afecta a su rendimiento profesional. Como último síntoma, según doctrina especialidad, destaca la despersonalización, desarrollar actitudes y sentimientos negativos hacia las personas con las que se trabaja $^{20}$. Este síndrome del burnout se considera el paso previo para que las consecuencias del estrés laboral tengan afectaciones en la salud del empleado a nivel físico, tales como alteraciones cardiorrespiratorias, fuertes dolores de cabeza, úlceras estomacales, insomnio o vértigos.

19 Según el cual: "Todas las prestaciones económicas que tengan su causa en accidente de trabajo o enfermedad profesional se aumentarán, según la gravedad de la falta, de un 30 a un 50 por ciento, cuando la lesión se produzca por equipos de trabajo o en instalaciones, centros o lugares de trabajo que carezcan de los medios de protección reglamentarios, los tengan inutilizados o en malas condiciones, $o$ cuando no se hayan observado las medidas generales o particulares de seguridad y salud en el trabajo, o las de adecuación personal a cada trabajo, habida cuenta de sus características y de la edad, sexo y demás condiciones del trabajador. La responsabilidad del pago del recargo establecido en el apartado anterior recaerá directamente sobre el empresario infractor y no podrá ser objeto de seguro alguno, siendo nulo de pleno derecho cualquier pacto o contrato que se realice para cubrirla, compensarla o trasmitirla. La responsabilidad que regula este articulo es independiente y compatible con las de todo orden, incluso penal, que puedan derivarse de la infracción».

20 Consultar: Gil-Monte, Pedro (2003). 


\section{Conclusión}

En atención a lo analizado, se puede afirmar que el nuevo derecho digital se superpone a un derecho ya conquistado y asentado como es el de los descansos legales, porque siempre ha existido el derecho a no estar disponible fuera del tiempo de trabajo. Aunque ahora, debido a la conectividad constante del s. xxI, el legislador ha incidido en esta cuestión y ha regulado sobre este respecto, quizás más como medidas de protección y prevención frente a la fatiga informática. Igualmente, ya existía el deber empresarial de proteger al trabajador frente a los riesgos laborales conforme a la LPRL.

Es lógico pensar que este derecho laboral incipiente en el ordenamiento jurídico interno, adquiere un especial significado en las actuales relaciones laborales porque con él, se limita el uso de las tecnologías y se garantiza todo lo concerniente al tiempo de trabajo (jornada laboral, horarios, fiestas y permisos, así como vacaciones). Una limitación del uso que resulta destacable, dado que las herramientas tecnológicas que están asentadas en las empresas desde hace años, van a tener un auge imparable y se van a potenciar exponencialmente. Indudablemente, mediante estas prestaciones de trabajo virtuales, se potencia la utilización de las herramientas tecnológicas, sobre todo la concerniente al uso de los teléfonos móviles inteligentes (smartphones) que provocan una conectividad constante tanto a través de llamadas telefónicas como de aplicaciones de mensajería instantánea como de correos electrónicos profesionales.

Como se ha puesto de relieve, las nuevas tecnologías han transformado, por completo, la manera de trabajar y gestionar los recursos en las empresas. Son clave para la optimización de tiempos y para la productividad. Es un hecho que las empresas cuentan con Internet en las oficinas físicas y, por consiguiente, que los trabajadores la utilicen para buscar información, para comunicarse o, incluso, para aprender (la formación on-line es muy potente). Y no solo en las oficinas físicas, en los hogares también, pues muchas empresas, aparte de dotar a sus trabajadores de dispositivos portátiles de carácter profesional (ordenadores -portátiles y de sobremesa-, smartphones, tablets, etc.) cuentan con trabajadores que realizan su actividad, de forma regular, fuera de sus locales. Para poder trabajar, se conectan a las TIC de sus empresas por medio de redes telemática (es común la utilización de conexión $V P N^{21}$, por ejemplo). Esta transformación digital que se instaura en el día a día de las empresas no ha de ir en perjuicio del respeto de los descansos legales de los trabajadores. En un futuro muy cercano el vigente derecho a la «desconexión digital» va a tener una relevancia precisamente porque la transformación digital de las empresas es inmensa; en tiempos de CO-

${ }^{21}$ Siglas de «Virtual Private Network» que significa red privada virtual; la utilizan muchas compañías como una tecnología de red de ordenadores que permite una extensión segura de la red de área local sobre una red pública o no controlada como Internet. 
VID-19 se potencia más si cabe este uso digital en el trabajo por la falta de actividad en lugares presenciales.

Durante la pandemia mundial, muchas empresas en España se han despreocupado de la salud mental y bienestar de sus teletrabajadores. Se ha dado así una vuelva de tuerca más a la ya elevada precariedad laboral del país. Parece que la mentalidad de buena parte del empresario está fijada en mantener la desconfianza en los propios trabajadores y someterlos a la dictadura de la vigilancia y control constantes — ahora también usando softwares informáticos dirigidos a controlar cada movimiento del trabajador-. En lugar de confiar en el trabajador, cediéndole autonomía y libertad para gestionar su propio tiempo, se le somete a una carga abusiva de trabajo a fin de asegurar un rendimiento ininterrumpido. De un presentismo laboral se está pasando a un "telepresentismo» o "presentismo digital». La precariedad y el control se ha trasladado ahora al ámbito cotidiano.

Más cuando los trabajadores que ya están agotados física y mentalmente del estrés causado por las tecnologías, en muchas ocasiones, por miedo a perder su empleo y aferrarse a su puesto de trabajo con lo que, a la postre, terminan unas tareas que no deberían desempeñar fuera de su horario y, obviamente, gratuitamente al no computarse como horas extraordinarias y, obviamente, en perjuicio de su salud. Esto es especialmente relevante, máxime cuando en España existe una alta temporalidad debido a la estacionalidad propia de sectores con mucho peso como es el del turismo. En este sentido, ante necesidades económicas de parte de la población (hipoteca, gastos familiares, etc.) si la gran mayoría de contratos firmados son contratos temporales las reclamaciones laborales de los trabajadores se envainan por temor a que no se les renueven sus contratos. Esta circunstancia es conocida por muchas empresas y de ahí que abusen de estos tipos de contratos laborales (no llegan a sobrepasar los límites ex. art. 15.5 ET).

Para el trabajador, es necesario un tiempo de descanso y de desconexión, que, en muchas ocasiones, en una sociedad en la que se tiende al trabajo en remoto y a la conciliación - más ahora con motivo del COVID-19—, da la sensación de que todo ello es incompatible con el derecho a la desconexión digital. Este derecho es más que necesario en la actual era tecnológica; muy pocos trabajadores logran desconectarse de su trabajo una vez que se termina la jornada. Ello provoca que se encuentren constantemente recordando tareas pendientes, pensando en las tareas del día siguiente, etc. lo cual genera una ansiedad y estrés constantes. Si a esto, se les suma la conectividad continua a correos electrónicas, SMS, mensajes instantáneos al móvil o llamadas del jefe tras la jornada laboral; jamás se descansa del trabajo. Se ha de garantizar el descanso pues, en caso contrario, se abocan a los trabajadores a un mayor agotamiento psicológico y laboral.

En efecto, merced a la LPRL la empresa debe velar para que se garanticen los derechos de los trabajadores tocantes a su seguridad y salud; el estrés oca- 
sionado por la no «desconexión digital» constituye un evidente riesgo psicosocial importante, por lo que las empresas han de sensibilizarse y tomar conciencia de ello en aras a adoptar medidas de prevención y protección.

A nivel empresarial existen aplicaciones ideadas por empresas especializadas que pueden ayudar a garantizar el desenganche de sus trabajadores $y$, de paso, dar cumplimiento a la normativa de aplicación. Un ejemplo es el de la empresa Fuifi $i^{22}$ la cual, ha creado lo según señalan desde su web: «la solución para la desconexión digital en tu empresa». Funciona en forma de app disponible en web mobile, app android y app iPhone, por la que, entre otras funcionalidades como son el sistema del registro de la jornada laboral —adaptado al Real Decreto-Ley 8/2019 de 8 de marzo 2019- y la gestión de vacaciones, permite que los trabajadores desconecten de sus tareas laborales. Así según detalla la app, los trabajadores pueden solicitar directamente desde la aplicación móvil el periodo de tiempo en el que van a estar de vacaciones, así como gestionar cualquier otra ausencia; indicando la fecha de inicio y fin y contabilizando el computo de horas totales que van a faltar. Por otro lado, en el caso de tener que atender alguna urgencia en el trabajo, con la misma app podrán ponerse en contacto con el resto de sus compañeros, si fuera necesario. Finalmente, para garantizar el ejercicio del derecho a la desconexión digital, permite que el trabajador pueda configurar sus periodos de descanso para no recibir notificaciones de la aplicación en ese tiempo.

Desde este prisma, las empresas deberían plantearse las consecuencias que tiene para la organización que una persona sufra semejante estrés laboral durante un periodo prolongado de tiempo; el papel de la negociación colectiva es todavía deficitaria en este sentido por lo que urge un mayor tratamiento del derecho en los convenios colectivos que garanticen la seguridad y salud en el trabajo de los riesgos propios de no «desenchufarse» de los medios digitales en el trabajo. La calidad de vida laboral y el estado de salud física y mental de los trabajadores de una organización tiene repercusiones sobre esa organización, como por ejemplo mayor absentismo, menor productividad, menor calidad en el producto $y$, en definitiva, mayor gasto económico y menor ganancia.

No cabe duda que un trabajador descansado es un trabajador más productivo y menos propenso a coger bajas laborales (evitará sobrecargarse en el trabajo y evitará padecer el síndrome del trabajador quemado — burnout—) por lo que, los empresarios, deben tomar buena nota de lo aquí señalado e intentar adoptar medidas en este sentido para que el trabajador una vez acabe su jornada, pueda descansar mentalmente y disfrutar de los suyos, practicar deporte, etc. Máxime cuando ello, es un mandato constitucional: si se acude a la carta magna, en su artículo 40.2 se encomienda a los poderes públicos, como uno de los principios rectores de la política social y económica, velar por la seguridad e

22 Consulta en: https: //fuifi.com/ (Accedido a 5-10-2020). 
higiene en el trabajo y garantizar el descanso necesario, mediante la limitación de la jornada laboral, las vacaciones periódicas retribuidas y la promoción de centros adecuados.

\section{Bibliografía}

Alemán, Francisco (2017): «El derecho de desconexión digital: una aproximación conceptual, crítica y contextualizadora al hilo de la "Loi Travail N.o 2016-1088». Trabajo y derecho: nueva revista de actualidad y relaciones laborales, ISSN 2386-8090, N.o 30.

Altés, Juan Antonio y YagüE, Sergio (2019): El derecho a la desconexión digital en el trabajo. Dentro del manual EL FUTURO DEL TRABAJO: CIEN AÑOS DE LA OIT (COMUNICACIONES). XXIX Congreso Anual de la Asociación Española del Derecho del Trabajo y de la Seguridad Social. Editor: Ministerio de Trabajo, Migraciones y Seguridad Social, Subdirección General de Información Administrativa y Publicaciones. Colecciones: Informes y Estudios. General, 23.

Altés, Juan Antonio y YagüE, Sergio (2020): «A vueltas con la desconexión digital: eficacia y garantías de lege lata». Labos, Vol. 1, No. 2, pp. 61-87 / doi: https: //doi. org/10.20318/labos.2020.5539 EISSN 2660-7360 - http: //www.uc3m.es/labos;

Alegre, Manuel (2018): La prevención de los nuevos riesgos psicosociales y el derecho a la "desconexión digital». Gestión práctica de riesgos laborales: Integración y desarrollo de la gestión de la prevención, ISSN 1698-6881, N.․ 155, 2018, págs. 38-41.

Arrieta, Francisco Javier (2019): La desconexión digital y el registro de la jornada diaria en España como mecanismos para garantizar el descanso, la salud y el bienestar de los trabajadores digitales a distancia. Lan harremanak: Revista de relaciones laborales, ISSN 1575-7048, N.o 42, 2019 (Ejemplar dedicado a: Digitalización, conciliación y trabajo como mercancía/ Digitalizazioa, uztartzea eta lana merkatalgai gisa).

Biurrun, Fernando (2018): «El derecho a la desconexión digital, más cerca». Actualidad jurídica Aranzadi, ISSN 1132-0257, N. o 943, 2018, págs. 22-22.

CCOO Endesa (2020): «Solicitamos la regulación urgente del Trabajo Fuera de Oficina (TFO) en la actual situación de fuerza mayor». Recuperado de: https: //ccooendesa. com/sites/default/files/2020-05/200518\%20Regulaci\%C3\%B3nTFO\%20.pdf [Consulta a 11 de junio 2020].

Dosi, Giovanni y Virgillito, Maria Enrica (2019): «¿Hacia dónde evoluciona el tejido social contemporáneo? Nuevas tecnologías y viejas tendencias socioeconómicas». Revista Internacional del Trabajo, vol. 138 (2019), núm. 4, pág. 3.

Domingo, José y Salvador, Neus (2020): «Hiperconectividad digital y salud laboral». Diario La Ley, ISSN 1989-6913, N.o 9638.

GiL-Monte, Pedro (2003). «Síndrome de quemarse por el trabajo (Síndrome de Burnout) en profesionales de enfermería». Revista Eletrônica Interaçao Psy, (1), 19-33.

López LOURDEs (2018): «Vigilar ocultamente a los trabajadores en la empresa y la reciente doctrina del Tribunal Europeo de Derechos Humanos». Disponible en: https: // www.ga-p.com/wp-content/uploads/2018/07/vigilar-ocultamente-a-los-trabajadoresen-la-empresa-y-la-reciente-doctrina-del-tribunal-europeo-de-derechos-humanos.pdf (Accedido: 3-06-2020).

Mińarro Yanini, Margarita (2020). «El sistema de prevención de riesgos laborales como garantía de efectividad del derecho a la desconexión digital». En Rodríguez-Piñeiro 
Royo, M. y Todolí Signes, A. Vigilancia y control en el Derecho del Trabajo Digital (1. ${ }^{\mathrm{a}}$ ed., pp. 577-596). Cizur Menor (Navarra): Aranzadi-Thomson Reuters.

Molina, Cristóbal (2017): «Jornada Laboral y Tecnologías de la Infocomunicación: «desconexión digital», garantía al descanso». Temas laborales, núm. 138/2017.

Molina, Cristóbal (2019): "Del acoso moral («mobbing») al ciberacoso en el trabajo («network mobbing») viejas y nuevas formas de violencia laboral como riesgo psicosocial en la reciente doctrina judicial». Estudios financieros. Revista de trabajo y seguridad social: Comentarios, casos prácticos : recursos humanos, ISSN 1138-9532, N. ${ }^{\circ} .437-$ 438, 2019, págs. 143-165.

Rodríguez Fernández, María Luz y Pérez del Prado, Daniel (2017). «Economía digital: Su impacto sobre las condiciones de trabajo y empleo. Estudio de caso sobre dos empresas de base tecnológica». Disponible en: http: //fdialogosocial.org/public/ upload/2/23_FdS_Economia-digital-impacto-condiciones-trabajo-y-empleo_2017_ final.pdf (Accedido: 2-6-2020).

SÁnchez Lacuesta, Javier (2020), «Desconexión digital». Disponible en: http: //www. biomecanicamente.org/news/ibv/item/1809-levante-articulo-abril2020.html (Accedido a 5-10-2020).

Sebastián Olga y Del Hoyo, María Ángeles (2016): «La carga mental del trabajo». Instituto Nacional de Seguridad e Higiene en el Trabajo. Composición e impresión: Servicio de Ediciones y Publicaciones. INSHT Madrid ISBN: 84-7425-605-4, pág. 16. Disponible en: https: //www.insst.es/documents/94886/96076/carga+mental+de+trabajo/2fd91b55f191-4779-be4f-2c893c2ffe37 (Accedido a 2-6-2020).

Todolí, Adrián (2017): «El derecho a la desconexión digital ya existe. Nos venden humo si no se establecen sanciones claras para quien lo vulnere». Disponible en: https: // adriantodoli.com/2017/03/29/el-derecho-a-la-desconexion-digital-ya-existe-nos-venden-humo-si-no-se-establecen-sanciones-claras-para-quien-lo-incumpla/ (Accedido: 2-6-2020).

Torres, Bárbara (2020): «Sobre la regulación legal de la desconexión digital en España: valoración crítica». Revista Internacional y Comparada de Relaciones Laborales y Derecho del Empleo, ISSN-e 2282-2313, Vol. 8, N.o. 1, 2020, pág. 255.

Trujillo Pons, Francisco (2020): «La «desconexión digital» en el ámbito laboral». Colección Laboral núm. 264, Tirant lo Blanch, Valencia. 166 páginas. 25 septiembre 2020. ISBN: 978-84-1355-912-4.

Pérez de los Cobos y Orihuel, Francisco (2019): «Poderes del empresario y derechos digitales del trabajador». Trabajo y derecho: nueva revista de actualidad y relaciones laborales, ISSN 2386-8090, N.o. 59.

Rodríguez, Sarai (2018): La desconexión digital como límite al control empresarial de la jornada de trabajo. La revolución tecnológica y sus efectos en el mercado de trabajo: un reto del siglo xxi / coord. por Alicia Villalba Sánchez; Lourdes Mella Méndez (dir.), 2018, ISBN 978-84-9020-717-8, págs. 493-513.

Santamaría, Noemí (2018): Las Tecnologías de la Información y la Comunicación (TIC) (I): nuevas formas de organización del trabajo. Recuperado de: https: //www. insst.es/documents/94886/566858/ntp-1122w.pdf/baa93260-6840-4b9b-9abbb6980b7f8f71 [Consulta a 2 junio 2020]

VIDAL, Pere (2018): «La desconexión digital laboral es ya una realidad». Actualidad jurídica Aranzadi, ISSN 1132-0257, N.o 946, 2018, págs. 11-11.

Zamora, Santiago (2019): «Desconexión digital ¿novedad o anécdota?», Diario La Ley, ISSN 1989-6913, N. 9363. 